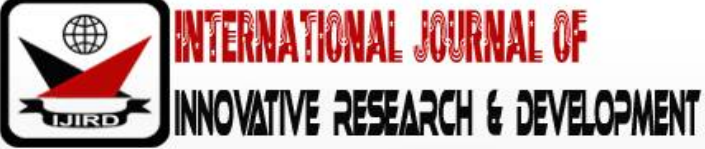

ISSN 2278 - 0211 (Online)

\section{Windowed Adaptive Filtering for Reducing Noise in Audio Signals during Transmission to Remote Locations}

\begin{tabular}{c}
\hline Afolabi M Taiwo \\
Student, Department of Electrical Electronics Engineering \\
Chukwuemeka Odumegwu Ojukwu University, Nigeria \\
Dr. Mbachu C B \\
Associate Professor, Department of Electrical Electronics Engineering \\
Chukwuemeka Odumegwu Ojukwu University, Nigeria \\
\hline
\end{tabular}

\begin{abstract}
:
Audio signals are so important to mankind nowadays that they are now carried over transmission lines to remote ends for entertainment purposes or to send messages across and other purposes. Incidentally these audio signals can be contaminated either at the source or along this transmission lines by unwanted signals such as Additive White Gaussian Noise (AWGN), Random Noise, power line noise and other noise signals. To preserve the integrity of the audio signals at the receiving end, any noise contaminating the signal must be reduced to the barest minimum. In this paper a finite impulse response filter based on LMS algorithm is developed to remove AWGN from audio signals. During the adaptation process of the filter, every updated coefficient sequence is modified with Blackman-Harris window before being applied to the noisy audio. After design the optimum sampling parameters of the filter are determined to be sampling frequency of $8000 \mathrm{~Hz}$, filter order of 29 and step size of 0.006. An audio signal is generated by loading the Hallelujah song in the M-file of matlab into work space. An AWGN is generated with the matlab and added to the generated audio signal to form a noisy or contaminated audio signal. When the noisy signal is applied to the designed filter result shows that the noise in the signal drastically reduced to give a clean audio signal. Listening to the uncontaminated, contaminated and filtered audio signals and comparing them confirms the effectiveness of the adaptive filter in audio signal processing.
\end{abstract}

Keywords: Blackman-Harris window, audio, noise, additive white Gaussian noise, adaptive filter

\section{Introduction}

With advancement in technology audio signals are now carried over transmission lines to remote ends for entertainment purposes, sending messages and other purposes. These transmission lines include mobile communication, multimedia communication and public address system transmission, lines. Incidentally these voice signals can be contaminated either at the source or along these transmission lines by unwanted signals such as additive white Gaussian noise (AWGN), random noise, power line noise and other noise signals.

For the integrity of the audio signals to be preserved at the receiving end, these noise signals must be removed or reduced drastically. In this work the interest is to remove the additive white Gaussian noise from audio during transmission. Additive white Gaussian noise adds itself to any noise that might be intrinsic in the information system, and has uniform power across the frequency band for the information system. In addition, it has normal distribution in the time domain with an average time domain value of zero. Several methods have been employed to remove the noise. One of such methods is by finite impulse response (FIR) digital filtering $[1,2,3,4,5]$. Another method is by infinite impulse response (IIR) digital filtering. The IIR digital filters demonstrated for this purpose by some researchers are (1) Butterworth $[6,7,8,9],(2)$ Chebyshev [6, 8] and (3) Elliptic [6, 8]. Also used by researchers to process audio signal is adaptive filtering [10].

One other powerful method for processing audio signal is wavelet transform [11,12, 13, 14, 15]. Descrete wavelet transforms which is a descretized form of wavelet transform was also employed by some researchers to denoise voice signal [16]. A related method of wavelet transforms and adaptive filtering was used to denoise speech signal by the authors in [17]. Other methods of audio signal processing are spectral subtraction [18] and block thresholding algorithm [19].

In terms of adaptive digital filtering technique of processing audio signals, some researchers have made meaningful contributions. In [20] the authors used LMS-based adaptive filter to cancel noise in audio signals. In [10] the authors modified recursive least square (RLS) algorithm to effectively cancel noise in speech signals.

Whereas many previous researchers have used various windows to design static FIR filters for audio signal processing, no researcher has been confirmed to have used Blackman-Harris window on adaptive filters for processing audio signals. 
Therefore, in this work Blackman-Harris window is used to design an adaptive FIR filter for removing additive white Gaussian noise (AWGN) from audio signal.

\section{Design of FIR Adaptive Filter with Blackman-Harris Window}

Fig. 1 depicts Blackman-Harris window while the mathematical model is shown as equation (1). A sampling frequency of $8000 \mathrm{~Hz}$, filter order of 29 and step-size of 0.006 are used in the design of the adaptive filter. The adaptive algorithm for the filter implementation is LMS adaptive algorithm. The coefficient update equation for LMS adaptive algorithm is presented as equation (2).

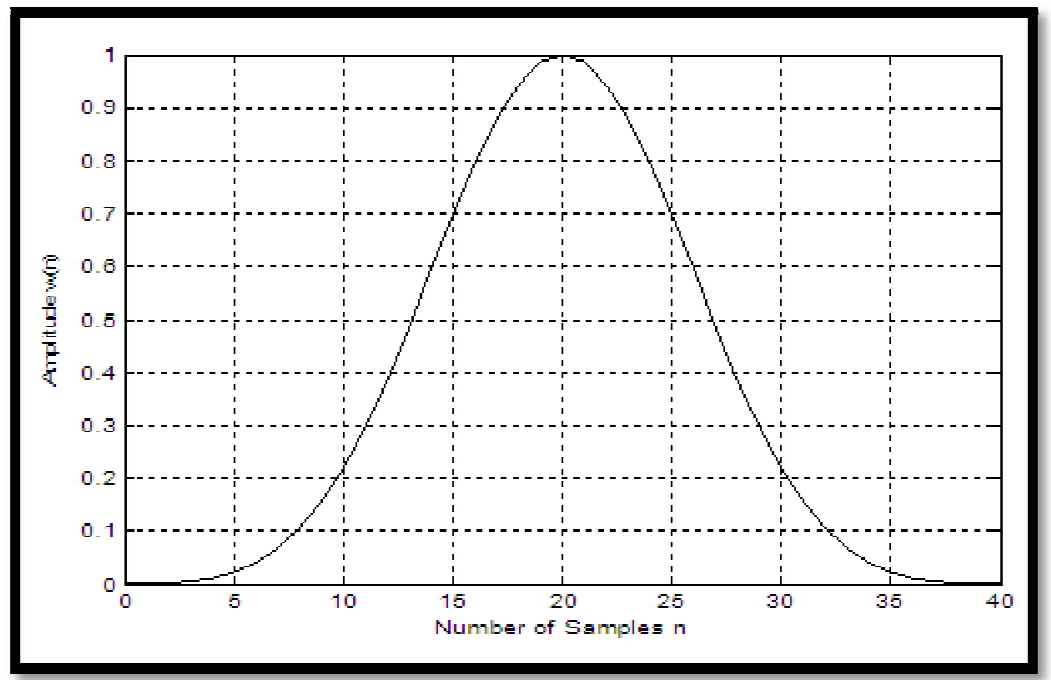

Figure 1: Blackman-Harris Window Function

$\mathrm{w}(\mathrm{n})=0.35875-0.48829 \cos \left(\frac{2 \pi \mathrm{n}}{\mathrm{M}-1}\right)+0.14128 \cos \left(\frac{4 \pi \mathrm{n}}{\mathrm{M}-1}\right)-0.01168\left(\left(\frac{6 \pi \mathrm{n}}{\mathrm{M}-1}\right)\right.$
$0 \leq \mathrm{n} \leq \mathrm{M}-1$

Where $\mathrm{M}$ is the number of samples and $\mathrm{n}$ varies from zero to number of samples.

$h(n+1)=h(n)+2 \mu e(n) x(n-k)$

Where $\mu$ is the step-size, $e(n)$ the error signal and $\mathrm{x}(\mathrm{n})$ is the primary input signal. Also, the $\mathrm{h}(\mathrm{n}+1)$ is the updated coefficient sequence while $h(n)$ is the previous coefficient sequence. Fig2. shows the adaptive noise canceller for removing noise from a desired signal. The primary input is the desired signal or contaminated signal while the secondary input is the noise signal to be removed.

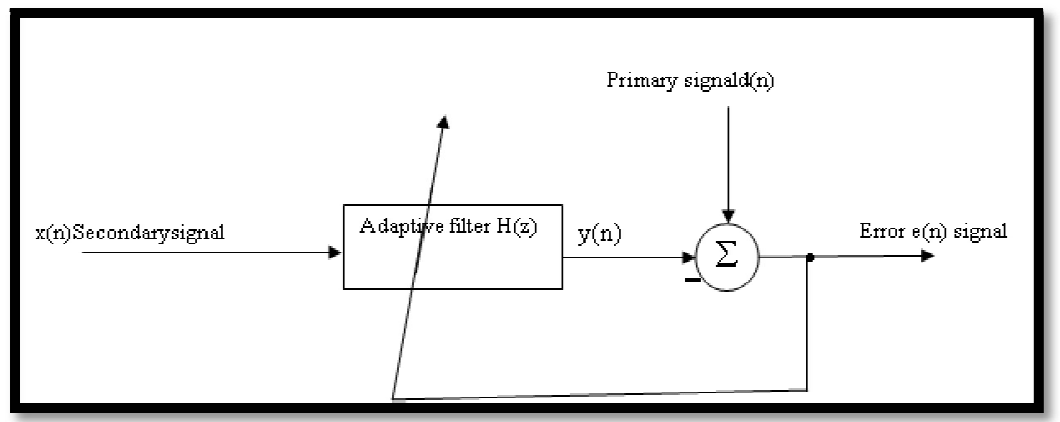

Figure 2: Adaptive Noise Canceller

The transfer function of adaptive filter is the same with that of a static filter, as presented in equation (3), except that the coefficient sequence varies during adaptation until convergence is attained.

$\mathrm{H}(\mathrm{z})=\mathrm{h}(0)+(\mathrm{h} 1) \mathrm{z}^{-1}+(\mathrm{h} 2) \mathrm{z}^{-2}+(\mathrm{h} 3) \mathrm{z}^{-3}+(\mathrm{h} 4) \mathrm{z}^{-4}+(\mathrm{h} 5) \mathrm{z}^{-5}+\ldots \ldots+\mathrm{h}(\mathrm{k}) \mathrm{z}^{\mathrm{L}}$

Where $h(n)$ is the impulse responseof the filter or the filter coefficients. With the design parameters of filter order, stepsize and sampling frequency stated above and based on LMS algorithm, the object of the filter is created with matlab. Based on the object, the instantaneous responses of impulse, magnitude, phase and z-domain of the filter are generated and depicted as fig.3, fig. 4, fig. 5 and fig. 6 respectively. 


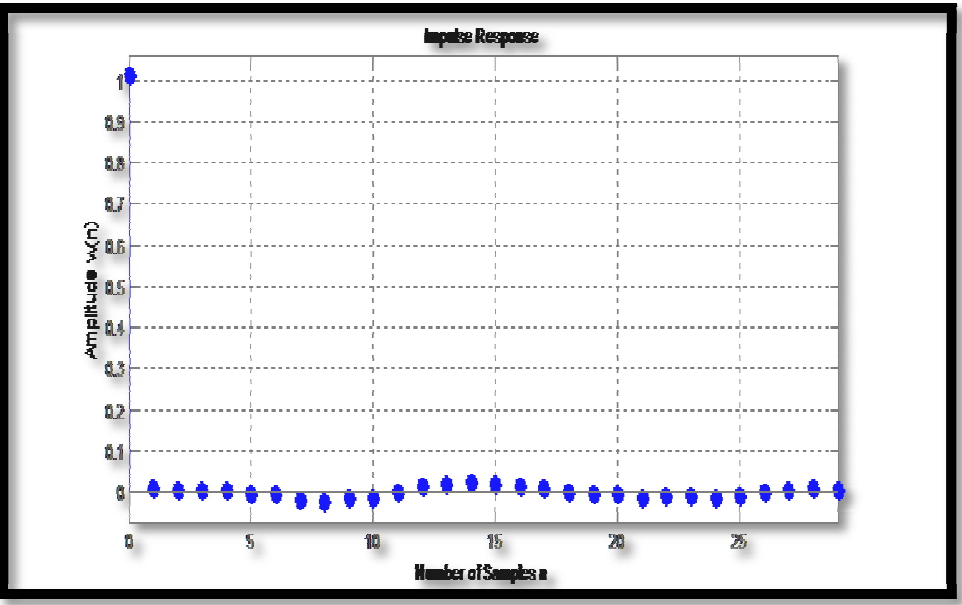

Figure 3: Impulse Response of the Filter

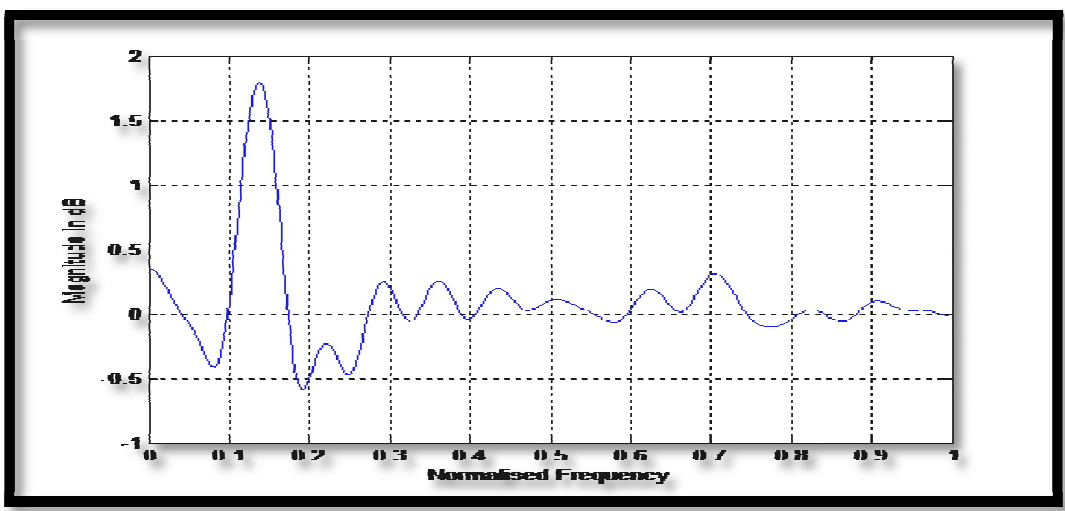

Figure 4: Magnitude Response of the Filter

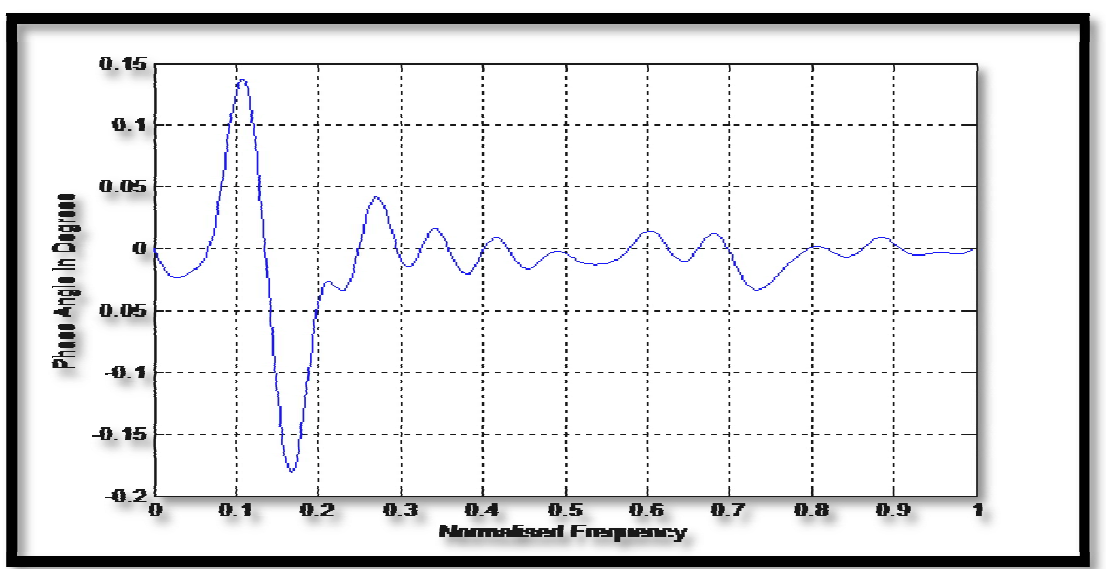

Figure 5: Phase Response of the Filter

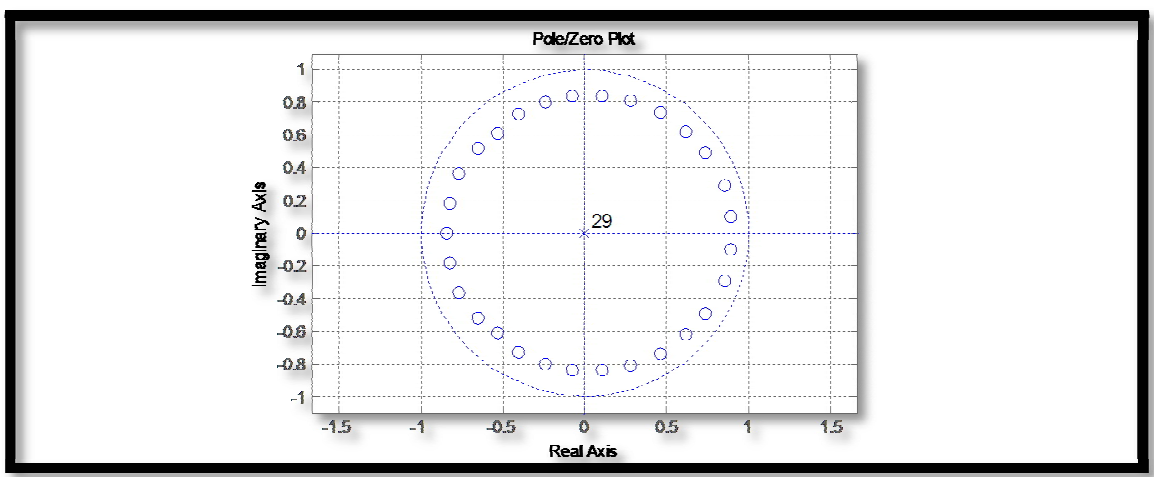

Figure 6: z-Domain Response of the Filter

The instantaneous impulse response of fig. 3 shows the amplitude of the response collapses to zero as the number of samples approaches the maximum value of 29 , implying that the filter is stable. The instantaneous response in $\mathrm{z}$-domain 
of fig. 6 shows that all the zeros are in alignment and all the poles and zeros are confined within the unit circle which indicates stability of the filter. The magnitude response of fig. 4 does not depict any sustained oscillation and this also means that the filter is stable. The phase response of fig. 5 depicts good linearity in phase.

\section{Structural Realization of the Designed Filter}

Based on these optimal parameters, filter order, step-size and sampling frequency of $29,0.006$ and $8000 \mathrm{~Hz}$ respectively, the proposed filter coefficients after convergence, at the end of update process are generated by simulation using Matlab as depicted in table 1 below. The transfer function of the designed filter deduced from table 1 is shown in equation (4) as;

$\mathrm{H}(\mathrm{z})=1.0111+0.0079 \mathrm{z}-1+0.0043 \mathrm{z}^{-2}+0.0049 \mathrm{z}^{-3}-0.0015 \mathrm{z}^{-4}+0.0057 \mathrm{z}^{-5}+0.0003 \mathrm{z}^{-6}$ $.0 .0129 z^{-28}+0.0112 z^{-29}$

$\mathrm{n}=$ Number of samplesh $(\mathrm{n})=$ Filter Coefficients

\begin{tabular}{|c|c|c|c|c|c|c|c|c|c|c|}
\hline $\mathbf{N}$ & $\mathbf{0}$ & & $\mathbf{1}$ & $\mathbf{2}$ & $\mathbf{3}$ & $\mathbf{4}$ & $\mathbf{5}$ & $\mathbf{6}$ & $\mathbf{7}$ & $\mathbf{8}$ \\
\hline $\mathrm{h}(\mathrm{n})$ & 1.0111 & & 0.0079 & 0.0043 & 0.0049 & -0.0015 & -0.0057 & -0.0073 & -0.0168 & -0.0165 \\
\hline $\mathrm{N}$ & 9 & & 10 & 11 & 12 & 13 & 14 & 15 & 16 & 17 \\
\hline $\mathrm{h}(\mathrm{n})$ & -0.0079 & & -0.0032 & 0.0105 & 0.0154 & 0.0134 & 0.0138 & 0.0081 & -0.0001 & -0.0041 \\
\hline $\mathrm{N}$ & 18 & & 19 & 20 & 21 & 22 & 23 & 24 & 25 & 26 \\
\hline $\mathrm{h}(\mathrm{n})$ & -0.0130 & & -0.0134 & -0.0076 & -0.0075 & -0.0022 & 0.0003 & 0.0017 & 0.0078 & 0.0084 \\
\hline $\mathrm{N}$ & 27 & & 28 & 29 & & & & & & \\
\hline $\mathrm{h}(\mathrm{n})$ & 0.0159 & & 0.0129 & 0.0112 & & & & & & \\
\hline
\end{tabular}

Table 1: Filter Coefficients after Convergence

Realization technique is the form the hardware components such as adders, delay elements, multipliers, etc. will be arranged in order to implement the system. Direct form is used in this research. In the structure, $\mathrm{h}_{0}, \mathrm{~h}_{1}, \mathrm{~h}_{2}, \mathrm{~h}_{3}, \mathrm{~h}_{4}, \ldots \ldots . . . \mathrm{h}_{29}$ are filter coefficient multipliers, while $\mathrm{z}^{-1}, \mathrm{z}^{-2}, \mathrm{z}^{-3}, \mathrm{z}^{-4}, \mathrm{z}^{-5}, \ldots \ldots . \mathrm{z}^{-29}$ are delay elements.

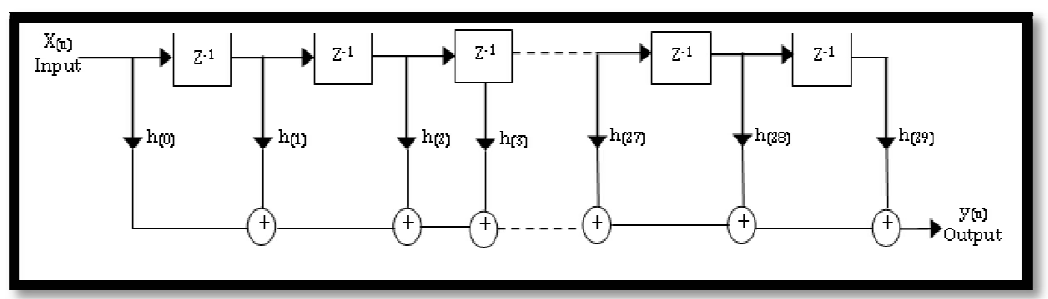

Figure 7: Structural Realization Diagram of the Filter

\section{Results}

A clean audio signal is generated with matlab program by load handle command. This command loads Handel's Hallelujah Chorus into the work space. This song is represented as fig.8. An additive white Gaussian noise is also generated with matlab and the signal is presented as fig.9. A contaminated audio signal is generated with matlab by the awgn command in matlab and the generated signal is depicted as fig.10. The contaminated audio signal of fig.10 is applied to the designed adaptive filter and the filtered output is shown in fig.11. The power spectral densities of the uncontaminated, contaminated and the filtered audio signals are presented in fig.12, fig.13 and fig.14 respectively.

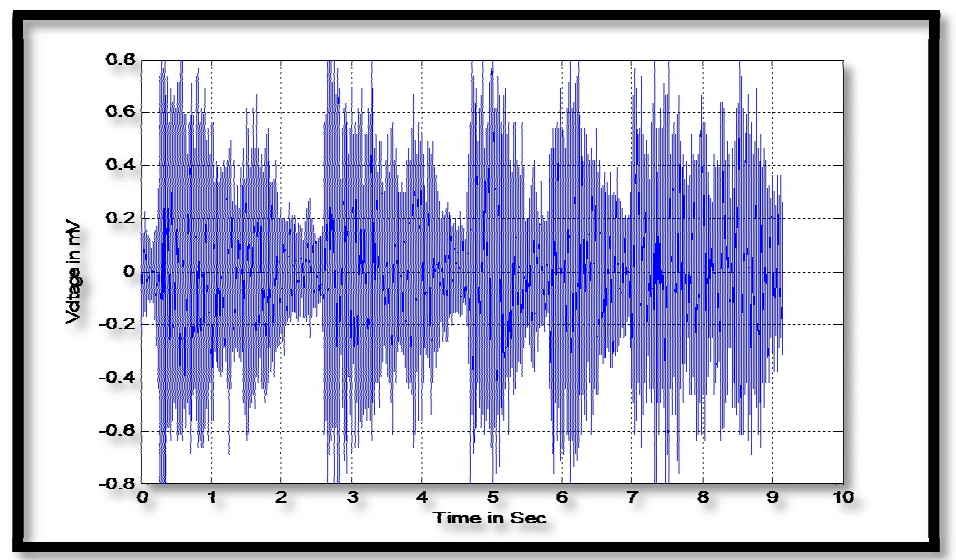

Figure 8: Uncontaminated Audio Signal 


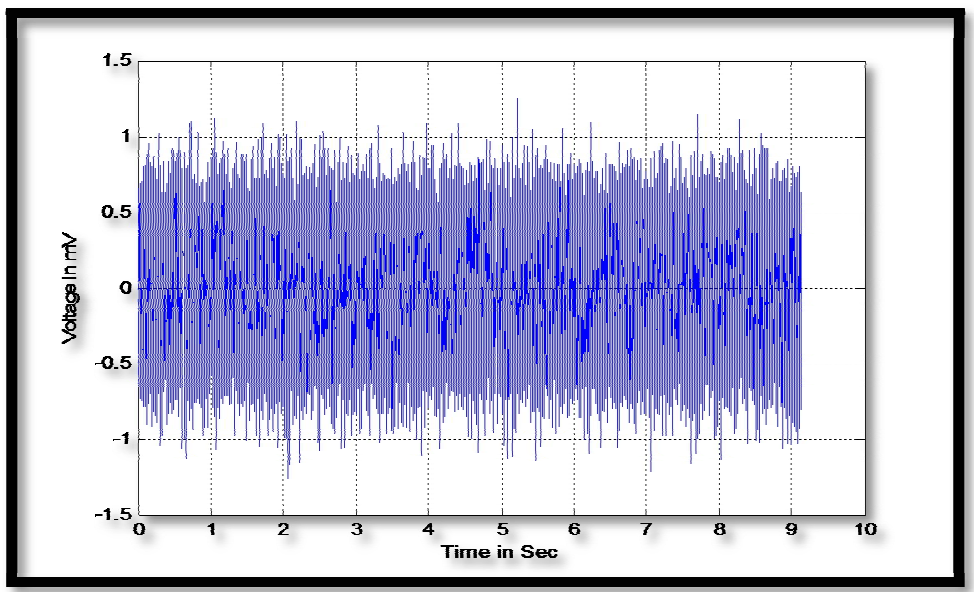

Figure 9: Additive White Gaussian Noise

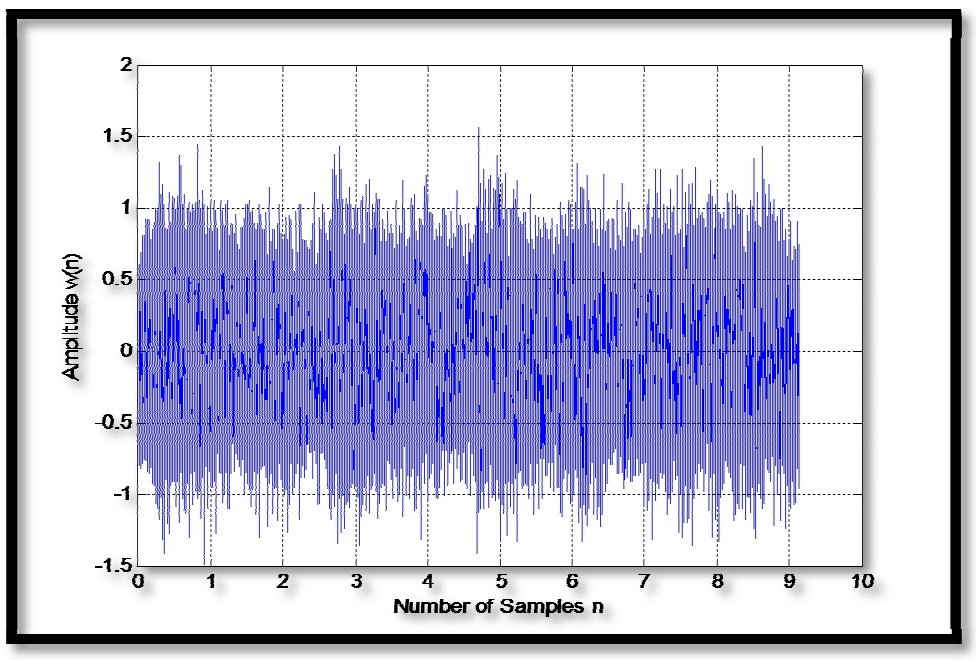

Figure 10: Contaminated Audio Signal

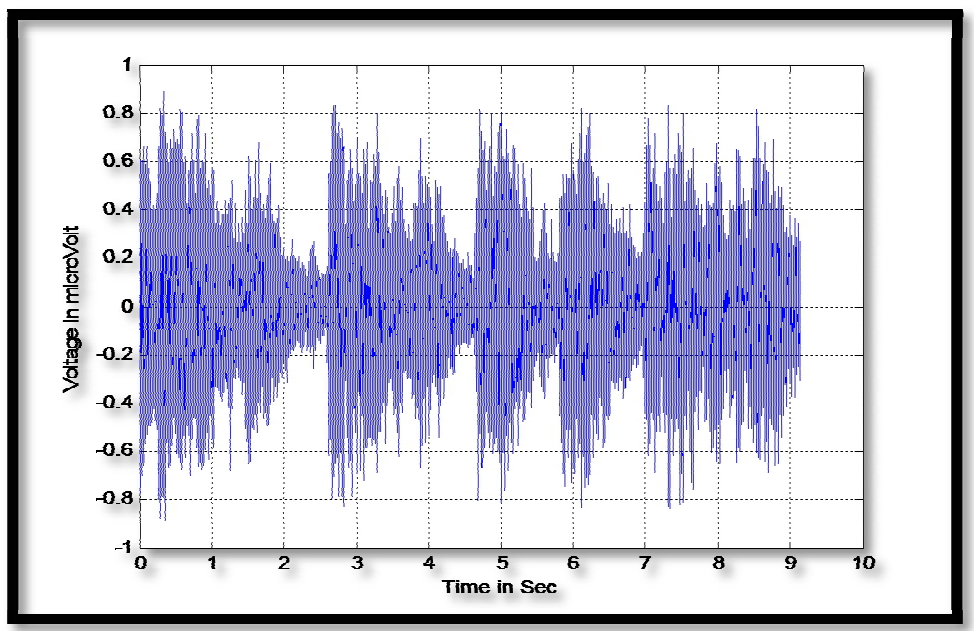

Figure 11: Filtered Audio Signal 


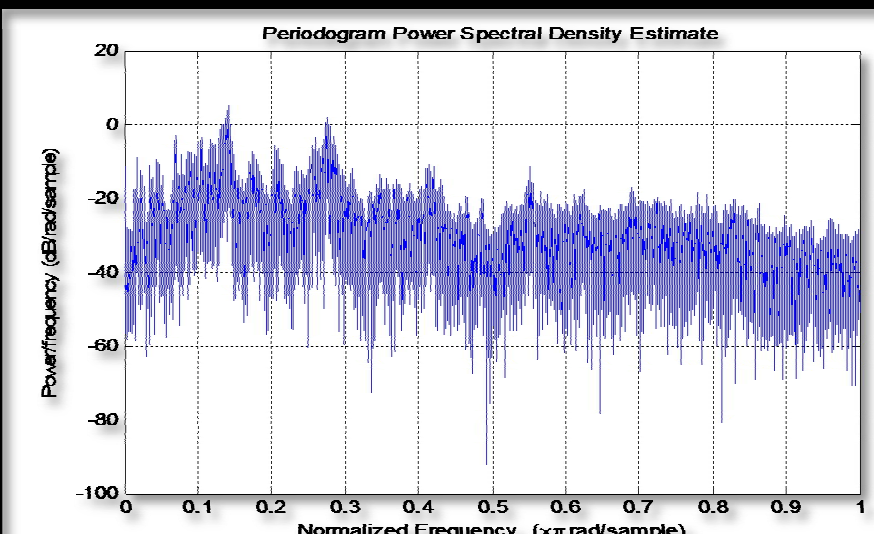

Figure 12: Power Spectral Density of the Uncontaminated Audio Signal

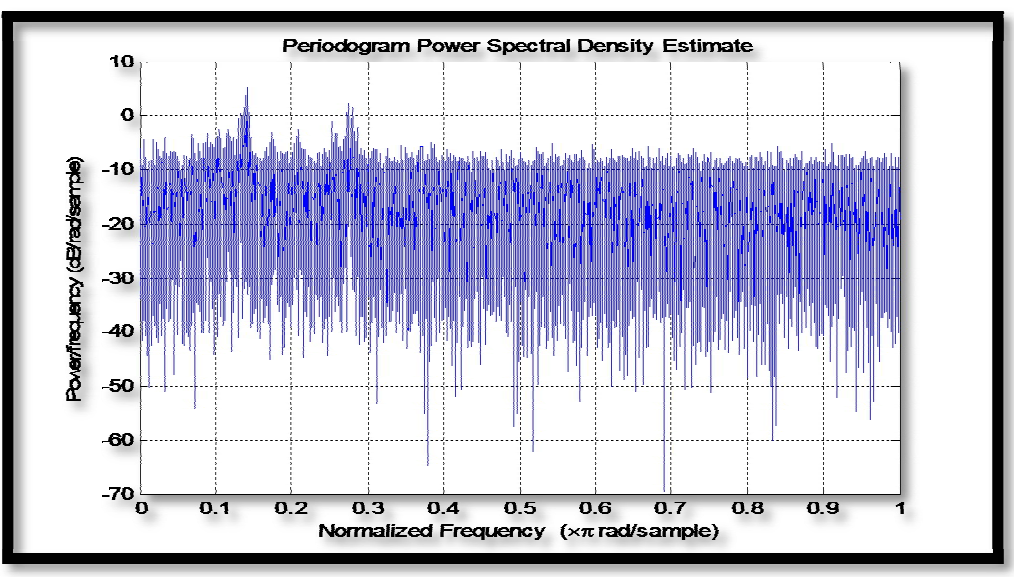

Figure 13: Power Spectral Density of the Contaminated Audio Signal

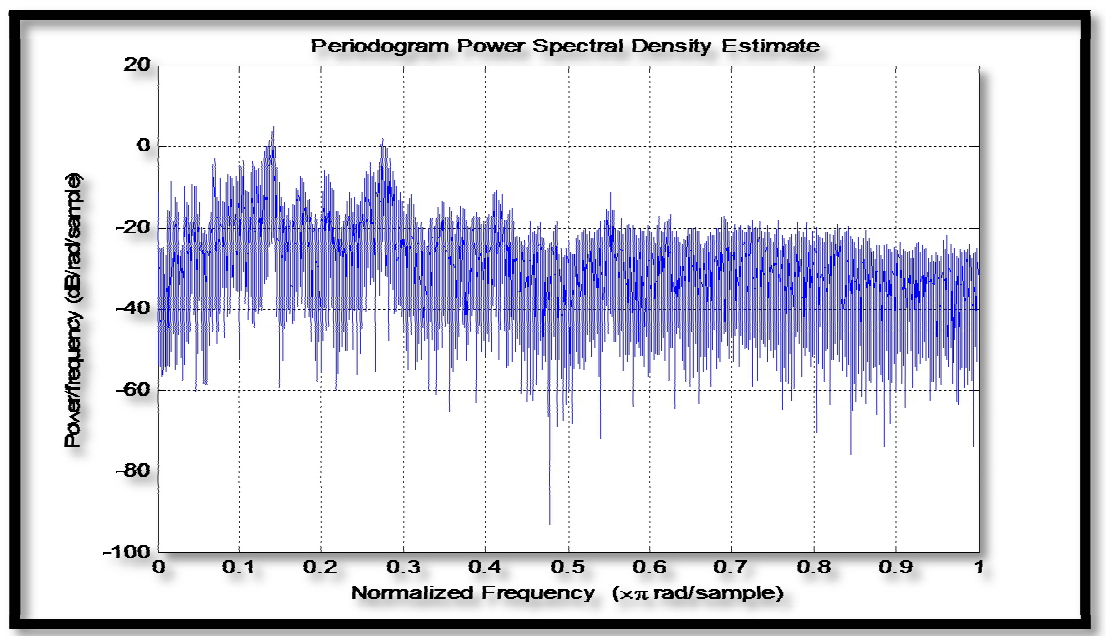

Figure 14: Power Spectral Density of the Filtered Audio Signal

By examining the uncontaminated audio signal of fig. 8, contaminated audio signal of fig. 10 and the filtered audio signal fig. 11, it is very clear that the filter drastically reduced the noise in the audio signal. From the power spectral densities of the uncontaminated, contaminated and filtered audio signal as depicted in figures 12,13 and 14 respectively, the signal and noise powers can be deduced as summarized in table 2 below. Only two noise points are chosen for demonstration, at normalized frequencies of 0.5 and 0.65 .

\begin{tabular}{|c|c|c|}
\hline Normalized Frequency Points & 0.5 & 0.65 \\
\hline Power Level of Clean Audio Signal in dB & -28.05 & -22.71 \\
\hline Power Level of Noisy Audio Signal in dB & -7.505 & -7.676 \\
\hline Power Level of Filtered Audio Signal in dB & -25.96 & -20.48 \\
\hline Noise Power Present in Noisy Audio Signal in dB & -20.545 & -15.034 \\
\hline
\end{tabular}

Table 2: Audio Signal and Noise Powers 
From table 2 it can be seen that the noise present in the noisy audio reduced the signal powers to -7.505dB from $28 \mathrm{~dB}$ at a frequency of 0.5 and to $-7.676 \mathrm{~dB}$ from $-22.71 \mathrm{~dB}$ at a frequency of 0.65 . When the filter is used on the noisy signal, signal power rises to $-25.96 \mathrm{~dB}$ at 0.5 normalisedfrequency and -20.48 at 0.65 normalized frequency; implying that the filter substantially degraded the noise.

\section{Conclusion}

This research has established the satisfactory performance of FIR adaptive filter modified with Blackman-Harris window function in audio signal processing.

In the filtration analysis the filter significantly removed the additive white Gaussian noise from the audio signal as can be seen in comparing the filtered audio of fig. 8 with the uncontaminated audio signal of fig. 11. Listening to the uncontaminated, contaminated and filtered audio signals in as matlab environment confirms the satisfactory performance of the filter in noise removal.

\section{References}

i. Rajput, S.S. \&Bhaduaria S.S. (2012). Implementation of FIR Filter Using Efficient Window Function and its Application in Filtering a Speech signal. International Journal of Electrical, Electronic and Mechanical Controls, 1(1).

ii. Elamaran, V., Upadhyay, H.N., Raju, N. \& Narasimhan, K. (2015). Real time Audio Denoising Using Digital FIR Filters with FPGA Implementation. International Journal of Pharmacy and Technology, 7(3), 9802-9810.

iii. Babu, P.S., Reddy, D.S. \& Reddy P.V.N. (2015). Speech Signal Analysis Using Windowing Techniques. International Journal of Emerging Trends in Engineering Research, 3(6), 257-263.

iv. [Saseendran, T.K. \&Mehra, R. (2014). Kaiser Window Based 21 Tap FIR Filter for Audio Application. International Journal of Advanced Engineering Research and Technology (IJAERT), 99-103. ISSN: 2348- 8190.

v. Pawar, R.\&Mehra R. (2014). Design and Performance Analysis of FIR Filter for Audio Application. International Journal of Scientific Research in Engineering and Technology (IJSRET). ISSN: 2278-0882; 122-126.

vi. Singla, M. \&Singh, H. (2015). Paper on Frequency Based Audio Noise Reduction Using Butterworth, Chebyshev and Elliptical Filter. International Journal on Recent and Innovation Trends in Computing and Communication, 3(10), 5989-5995.

vii. Singh, M. \&Garg, N.K. (2014). Audio Noise Reduction Using Butterworth Filter. International Journal of Computer and Organization Trends, 6(1), 20-23.

viii. Podder, P., Hasan, M. and Islam, R. (2014). Design and Implementation of Butterworth, Chebyshev and Elliptic Filter for Speech Signal Analysis. International Journal of Computer Applications, Vol.98, No.7, pp. 12-18.

ix. Chawaldhal, S.R., Khuje, P.S., Pise, V.K., Barbuddhe, V.K. \&Shelotkar, A.D (2017). International journal of Advanced Innovative Technology, 2(2), 61-64.

x. Vijaykumar, V. R. and Vanathi, P. T. (2014). Modified Adaptive Filtering Algorithm for Noise Cancellation in Speech Signals. Journal of Electrical and Electronic Engineering, Vol. 2(74), pp. 17-20

xi. Kumara, V.S.R. \&Devarakonda, D.K (2013). A Wavelet Based Denoising of Speech Signal. International Journal of Engineering Trends and Technology, 5(2), 107-115.

xii. Mesharam, V. \&Swami P.D. (2016). Denoising of Speech Signal by Classification into Voiced, Unvoiced and Silence Region.IOSR Journal of Electronics and Communication Engineering, 11(l), 26-35.

xiii. Lonare, T.A. \& Bachute, M.R. (2016). Speech Denoising Using Wavelet Transform. ISOR Journal of VLSI and Signal Processing, 6(3), 36-41.

xiv. Mihov, S.G., Ivanov, R.M. \& Popov, A.N. (2009). Denoising speech signals by wavelet transform. Annual journal of electronics, ISSN 1313-1842

xv. Sharma, K. \&Haksar, P. (2012). Speech Denoising and Speech Enhancement Using Wavelet Filter.IOSR Journal of Engineering, 2(l), 150-153.

xvi. Jashapreet, K., Seema and Sunil, K, (2016). Audio Noise Reduction Using Descrete

xvii. Wavelet Transform and Filter. International Journal of Advanced Research in Computer

xviii. Science and Technology, Vol.4, Issue 2, pp. 165-168.

xix. Renisha, G. \&Jayasree, T. (2015). Enhancement of Speech Signals in a Noisy Environment Based on Wavelet based Adaptive Filtering. International Journal of Signal Processing, Image Processing and Pattern Recognition, 8(9), 6976.

xx. Karam, M., Khazaal, H.F., Aglan, H. \&Cole, C. (2014). Noise removal in Speech Processing Using Spectral Subtraction.Journal of Signal and Information Processing, 5, 32-41.

xxi. Harini, V., Sindhu, B., Kumari, G.S. \&Khan, H. (2011). Block Thresholding Algorithm for Enhancement of an Audio Signal Corrupted by Noise. International Journal of Research and Reviews in Applied Sciences (IJRRAS), 7(2),213217.

xxii. Karne V. M. and Oad, S. (2014). Noise Cancellation in Voice Signal Using LMS Adaptive Filter. International Journal of Emerging Technology and Advanced Engineering, Vol. 4, Issue 10, Pp. 656-659. 